\title{
ON GENERATING FUNCTIONS OF CLASSICAL POLYNOMIALS
}

\section{ARUN VERMA}

ABSTRACT. An expansion of an arbitrary power series in terms of products of a power series and a polynomial $p_{n}^{(a, \beta)}(x)$ (where $a, \beta$ are not necessarily independent of $n$ ) is given.

1. Introduction. Generating functions of the polynomials like $p_{n}^{\alpha, \beta}(x)$ where $\alpha, \beta$ are not independent of $n$ has drawn the attention of some investigators like Toscano [10], Feldheim [5], Al-Salam [1], etc. But during the past two or three years there has been a great rush in generalizing and discovering new generating functions for such polynomial sets. On this score Brown [3], Carlitz [4], Srivastava [8], and Zeitlin [11] have proved generalizations of a single result due to Brown [2] in different directions. In this note it is intended to show that most of the results of Brown, Carlitz, Srivastava and Zeitlin follow as special cases of a single known result for generalized hypergeometric series. The note is concluded by proving a generalization of the aforesaid result involving generalized hypergeometric series.

2. Verma [9] had proved

$$
p+s F_{q}\left[\begin{array}{c}
\left(a_{p}\right),\left(b_{s}\right) ; x w \\
\left(c_{q}\right)
\end{array}\right]=h \sum_{n=0}^{\infty} \frac{[h-n \alpha+1]_{n-1}\left[\left(b_{s}\right)\right]_{n}\left[\left(e_{u}\right)\right]_{n}}{[1]_{n}\left[\left(c_{q}\right)\right]_{n}}(-x)^{n}
$$

$$
\begin{aligned}
& { }_{p+2} F_{u+2}\left[\begin{array}{c}
\left(a_{p}\right), 1+h(1-\alpha)^{-1},-n ; w \\
\left(e_{u}\right), h(1-\alpha)^{-1}, b-n \alpha+1
\end{array}\right] \\
& \cdot{ }_{s+u+1} F_{q}\left[\begin{array}{c}
\left(b_{s}\right)+n,\left(e_{u}\right)+n, h+n(1-\alpha) ; x \\
\left(c_{q}\right)+n
\end{array}\right],
\end{aligned}
$$

provided $p+s \leq q$ or $p+s=1+q$ and $|x w|<1 ; s+u+1<q$ or $s+u=$ $q$ and $|x|<1$, and the series of the hypergeometric functions on the

Received by the editors August 20, 1971.

AMS (MOS) subject classifications (1970). Primary 33A30.

Copyright $\odot$ 1974, American Mathematical Society 
right-hand side is absolutely convergent. (This transformation is a slight generalization of an earlier result due to Niblett [7].)

In the above transformation, as usual, $\left(a_{r, p}\right)$ means the sequence of $(r-p+1)$ parameters $a_{r}, a_{r+1}, \cdots, a_{p}$ (when $r=1$ write $\left(a_{p}\right)$ instead of $\left.\left(a_{1, p}\right)\right) ;[a]_{n}=a(a+1)(a+2) \cdots(a+n-1),[a]_{0}=1 ;[a]_{-n}=(-)^{n} /[1-a]_{n}$ and

$$
{ }_{r} F_{s}\left[\begin{array}{c}
\left(a_{r}\right) ; z \\
\left(b_{s}\right)
\end{array}\right]=\sum_{n=0}^{\infty} \frac{\left[\left(a_{r}\right)\right]_{n}}{\left[\left(b_{s}\right)\right]_{n}} \frac{z^{n}}{n !} ; \quad r \leq s \text { or } r=s+1 \text { and }|z|<1 .
$$

In the first instance if in (2.1) we replace $p, u, q$ and $s$ by $p+1, u+$ $1, s+u+2$ and $s+1$, respectively, and set $a_{p+1}=a /(1+b), b_{s+1}=c_{s+1}$ $=a /(1+b), e_{u+1}=1+a /(1+b),\left(c_{s}\right)=\left(b_{s}\right),\left(c_{s+2}, s+u+1\right)=\left(e_{u}\right), c_{s+u+2}=$ $1+a /(b+1), x=t$ and $w=x$, we get the following results due to Srivastava [8, Equation (9)]:

$$
\begin{aligned}
\sum_{n=0}^{\infty}\left(\begin{array}{c}
a+(b+1) n \\
n
\end{array}\right){ }_{p+1} F_{q+1}\left[\begin{array}{c}
\left(\alpha_{p}\right),-n ; x \\
\left(\beta_{q}\right), 1+a+b n
\end{array}\right] t^{n} \\
=\frac{(1+v)^{a+1}}{1-b v}{ }_{p} F_{q}\left[\begin{array}{c}
\left(\alpha_{p}\right) ;-x v \\
\left(\beta_{q}\right)
\end{array}\right],
\end{aligned}
$$

where $v=t(1+t)^{b+1} ; \quad(0)=0$.

Similarly specializing the parameters in (2.1) the other result of Srivastava [8, Equation (11)] can also be deduced as a special case. Hence the results of Feldheim [5], Carlitz [4] and Brown [2], [3] can be obtained as has been shown by Srivastava [8]. As a matter of fact some of the results of Al-Salam [1] and Toscano [10] can also be obtained as special cases of Srivastava's result (2.2). In particular, if in (2.2) we set $b=1$, replace $a$ by $a-1$, we get

$$
\begin{aligned}
\sum_{n=0}^{\infty} \frac{[a+n]_{n}}{n !}{ }_{p+1} F_{q+1}\left[\begin{array}{c}
\left(\alpha_{p}\right),-n ; x \\
\left(\beta_{q}\right), a+n
\end{array}\right] t^{n} & \\
& =\frac{1}{\sqrt{1-4 t}}\left(\frac{2}{1+\sqrt{1-4 t}}\right)^{a-1}{ }_{p} F_{q}\left[\begin{array}{c}
\left(\alpha_{p}\right) ; \frac{-4 x t}{(1+\sqrt{1-4 t})^{2}} \\
\left(\beta_{q}\right)
\end{array}\right],
\end{aligned}
$$

which is a slight generalization of a result due to Toscano [10].

However if in (2.2) we set $p=3, q=2, b=0, a_{1}=-r, a_{2}=\gamma+r+1$, 
$\alpha_{3}=1+a, \beta_{1}=1+\alpha, \beta_{2}=1+\beta$ and write $w /(1-w)$ for $t$ we get

$$
\begin{aligned}
\sum_{n=0}^{\infty}\left(\begin{array}{c}
a+n \\
n
\end{array}\right){ }_{3} F_{2} & {\left[\begin{array}{c}
-n,-r, \gamma+r+1 ; x \\
1+\alpha, 1+\beta
\end{array}\right] w^{n} } \\
& =(1-w)^{a-1}{ }_{3} F_{2}\left[\begin{array}{c}
-r, \gamma+r+1, a+1 ;-\frac{x w}{1-w} \\
1+\alpha, 1+\beta
\end{array}\right],
\end{aligned}
$$

which for $a=0$ reduces to a result due to Al-Salam [1, Equation (6.3)]. Similarly the transformations (6.12) and (6.11) of Al-Salam [1] can also be obtained by specializing the parameters in (2.2). (It may be worth mentioning that (6.12) could be deduced from (6.11) by replacing $w$ by $w / \lambda$ and letting $\lambda \rightarrow \infty$.)

On the other hand if in (2.1) we set $p=q=2, s=u=1, a=1-b, h=$ $c, w=1$ and $a_{1}=c-a, a_{2}=c / b, e_{1}=1+(c / b), b_{1}=c, c_{1}=c, c_{2}=1+$ $(c / b)$ and write $-x$ for $x$, then, on using the following transformation on the left-hand side of the resulting expression

$$
{ }_{2} F_{1}\left[\begin{array}{c}
a, b ; x \\
c
\end{array}\right]=(1-x)^{-a}{ }_{2} F_{1}\left[\begin{array}{c}
a, c-b ; \frac{x}{x-1} \\
c
\end{array}\right] \text {, }
$$

we get the following elegant transformation of Gould [6]:

$$
\begin{aligned}
& \sum_{n=0}^{\infty} \frac{c}{c+b n}\left(\begin{array}{c}
a+b n \\
n
\end{array}\right)\left[\frac{x}{(1+x)^{b}}\right]^{n} \\
& \quad=(1+x)^{a} \sum_{n=0}^{\infty}(-)^{n}\left(\begin{array}{c}
a-c \\
n
\end{array}\right)\left(\begin{array}{c}
c / b+n \\
n
\end{array}\right)^{-1}\left(\frac{x}{1+x}\right)^{n}
\end{aligned}
$$

However in the above transformation if we replace $a$ and $c$ by $a+b r$ and $c+b r$, respectively, and sum both the sides from $r=0$ to $\infty$ after multiplying both sides by $\left[\left(a_{p}\right)\right]_{r} x^{r} /\left[\left(b_{q}\right)\right]_{r}[1]_{r}$, we get, on some simplification, the theorem due to Zeitlin [11, Theorem II].

3. We conclude the note by proving the following generalization of (2.1).

$$
\sum_{n=0}^{\infty} c_{n} d_{n} \frac{x^{n} w^{n}}{n !}=\sum_{n=0}^{\infty} \frac{(-x)^{n}}{n !} \sum_{k=0}^{\infty}\left(\begin{array}{l}
n \\
k
\end{array}\right) c_{k}[h+k(1-\alpha)](-w)^{k}
$$

$$
\text { - } \sum_{s=0}^{\infty}\left[\left(e_{u}\right)+k\right]_{s+n-k}[h+k+1-n \alpha]_{s+n-k-1} d_{s+n} \frac{x^{s}}{[1]_{s}},
$$

where $\left\{c_{n}\right\}$ and $\left\{d_{n}\right\}$ are arbitrary sequences. 
Proof. Equating the coefficients of $w^{N}$ on both the sides we get $\frac{d_{N}}{N !}=\{h+(1-\alpha) N\} \sum_{r=0}^{\infty} \frac{(-)^{r}}{(r+N) !} \sum_{s=0}^{\infty} \frac{\left[\left(e_{u}\right)+N\right]_{r+s}[h+N-N \alpha-r \alpha+1]_{r+s-1}}{[1]_{s}} d_{N+r+s} x^{s+r}$.

The coefficient of the term independent of $x$ on the right-hand side is readily seen to be $d_{N} / N$ !. So it only remains to show that for $M>0$,

$$
\{h+(1-\alpha) N\} \frac{\left[\left(e_{u}\right)+N\right]_{M}}{[1]_{M}} d_{N+M} \sum_{r=0}^{M} \frac{[-M]_{r}[h+N-N \alpha-r \alpha+1]_{M-1}}{r !}=0,
$$

which follows readily from the fact that $[h+N-N \alpha-r a+1]_{M-1}$ is a polynomial in $r$ of degree $M-1$ and so its $M$ th difference vanishes. This completes the proof of (3.1).

(3.1) reduces to (2.1) if we set $C_{n}=\left[\left(a_{p}\right)\right]_{n} /\left[\left(e_{u}\right)\right]_{n}, d_{n}=\left[\left(b_{s}\right)\right]_{n} /\left[\left(c_{a}\right)\right]_{n}$. However it may also be mentioned that the result added in the proof by Srivastava [8], which is attributed to Professor Carlitz, could be deduced as a special case of (3.1).

\section{REFERENCES}

1. N. A. Al-Salam, A class of hypergeometric polynomials, Ann. Mat. Pura Appl. (4) 75 (1967), 95-120. MR $36 \# 435$.

2. J. W. Brown, On zero type of Laguerre polynomials, Duke Math. J. 35 (1968), $821-823$.

3. - New generating functions for classical polynomials, Proc. Amer. Math. Soc. 20 (1969), 263-268.

4. L. Carlitz, Some generating functions for Laguerre polynomials, Duke Math. J. 35 (1968), 825-827. MR 39 \#1700.

5. E. Feldheim, Relations entre les polynomes de Jacobi, Laguerre et Hermite, Acta Math. 74 (1942), 117-138. MR 7, 65.

6. W. H. Gould, A series transformation for finding convolution identities, Duke Math. J. 28 (1961), 193-202. MR 23 \#A1216.

7. J. D. Niblett, Some hypergeometric identities, Pacific J. Math. 2 (1952), 219-225. MR 13, 940.

8. H. M. Srivastava, Generating functions for Jacobi and Laguerre polynomials, Proc. Amer. Math. Soc. 23 (1969), 590-595. MR 40 \#2935.

9. A. Verma, $A$ class of generating functions of $G$-functions and the Laplace transform, Math. Comp. 19 (1965), 664-665.

10. L. Toscano, Funzioni generatrici di particolari polinomi di Laguerre e di altri da essi dipendenti, Boll. Un. Mat. Ital. (3) 7 (1952), 160-167. MR 14, 269.

11. D. Zeitlin, A new class of generating functions for hypergeometric polynomials, Proc. Amer. Math. Soc. 25 (1970), 405-412. MR $41 \# 8719$.

DEPARTMENT OF MATHEMATICS, UNIVERSITY OF ALBERTA, EDMONTON, ALBERTA, CANADA 